\title{
Risk of Gestational Diabetes Mellitus (GDM) Using Clinical Prediction Model Based on Maternal Characteristics
}

\author{
Jahan $\mathrm{S}^{1}$, Ali $\mathrm{M}^{2}$
}

\begin{abstract}
Introduction: The healthcare delivery challenges in Bangladesh are phenomenal. Improving maternal and child health, reducing the high maternal and infant mortality \& morbidity are challenging. Arrangement of additional expenditure for GDM screening is again challenging. The efficiency of screening could be enhanced by considering women's risks of gestational diabetes on the basis of their clinical characteristics.
\end{abstract}

Objectives: To find out the use of the clinical prediction model of gestational diabetes mellitus (GDM) is valid for Bangladeshi pregnant women and to assess the risk of gestational diabetes by using clinical prediction model based on maternal characteristics.

Materials and Methods: A cross sectional study was carried out from July 2011 to June 2012 among purposively selected 217 pregnant women of $\geq 24$ weeks of gestation in the Gynae and Obstetric outpatient department of Combined Military Hospital, Dhaka. Data were collected by face to face interview, anthropometric measurement and record review. Two step oral glucose tests were done for diagnosis of GDM.

Results: According to Chadakaran clinical prediction model $84(38.7 \%)$ respondents were at high risk, $92(42.4 \%)$ were at intermediate risk and $41(18.9 \%)$ found at low risk of gestational diabetes but only $24(11.05 \%)$ developed gestational diabetes. Highest occurrence of gestational diabetes was found in high risk group 17 (20.2\%) with zero occurrence in low risk group. Risk score performance at the level of $\geq 380$, sensitivity was $100 \%$ and specificity $21.8 \%, 13.6 \%$ positive predictive value, $100 \%$ negative predictive value and area under curve was 0.385 . At the level of 460 score the sensitivity and specificity was found closest ( $70.8 \%$ and $65.3 \%$, respectively) and area under curve was highest 0.657 . The receiver operating characteristics curve of the risk score in the study sample for predicting women with glucose tolerance test demonstrated an area 0.763 (95\%, 0.682 0.845).

Conclusion: The use of clinical prediction model is a simple, non invasive, cost effective useful method to identify women at increased risk of gestational diabetes mellitus and could be short listed for further testing.

Key-Words: Gestational Diabetes Mellitus (GDM), Clinical Prediction Model, Maternal Characteristics.

\section{Introduction}

Gestational diabetes mellitus is a substantial and growing health concern in many parts of the world. Certain populations are specially vulnerable to develop this condition because of genetic, social, and environmental factors. Gestational diabetes has serious, long-term consequences for both baby and mother, including a predisposition to obesity, metabolic syndrome, and diabetes later in life. Early detection and intervention can greatly improve outcomes for women with this condition and their babies. Unfortunately, screening and diagnostic tests are not uniform worldwide, which could lead not only to under diagnosis but also under management of the illness and most are in low- and middle-income countries. Community based studies in Bangladesh showed the prevalence of $\mathrm{GDM}^{1}$ is as $6.8 \%$ in rural area. But hospital based studies are yet limited. Over the past decade a growing prevalence of GDM has been observed in the hospital of Bangladesh. Increased maternal and peri-natal mortality may be a part of the effect of GDM.

1. Maj Sharmin Jahan, MBBS, MPH, M.Phil(PSM), DADMS, HQ 24 Infantry Division; 2. Brig Gen Mohammad Ali, MBBS, DPH, MPhil(PSM), Professor and Head of the Department of Community Medicine, AFMC, Dhaka. 
As per 'WHO' guidelines, the usual approach of detecting gestational diabetes mellitus is to screen all pregnant women by measuring their plasma glucose after a $75 \mathrm{gm}$ oral glucose load at 24 to 28 weeks' gestation ${ }^{2}$. Screening for gestational diabetes mellitus has been included in a routine antenatal care program in many parts of the world $^{3,4,5}$. However, considering the global demographic data that approximately 130 million women become pregnant annually, this practice would result in a large number of women requiring GDM screening ${ }^{6}$. Thus, it is important for health care providers to set up an effective but affordable screening program for this metabolic disorder. The efficiency of screening could be enhanced by considering women's risks of gestational diabetes on the basis of their clinical characteristics ${ }^{2}$. Various risk assessment tools have been developed to identify persons most in need of screening. Yet, many of these risk assessment tools were developed from specific cohorts, which often have restricted age ranges or racial or ethnic groups, limits the generalized approach to the entire population. The purpose of this study is to estimate risk for predicting women who are likely to have an abnormal Glucose Tolerance Test (GTT) using a clinical prediction model based on maternal characteristics and also to see what extent of the model is valid for Bangladeshi women. So that this model can be used in clinical practice and timely intervention can be taken for maternal and neonatal benefit and also to reduce unnecessary burden of screening on women and healthcare system.

\section{Materials and Methods}

This cross sectional study was carried out at the outpatient department (OPD) of Obstetric and Gynecology unit of Combined Military Hospital $(\mathrm{CMH})$, Dhaka which is one of the best tertiary level hospitals in Bangladesh. A fairly large number of pregnant women receive antenatal care from the Gynecology and Obstetric outdoor of this Hospital. Pregnant women of $\geq 24$ weeks of gestational age who had single pregnancy and had first trimester weight record came for routine antenatal checks up were included in the study. Twin or multiple pregnancy, known hypertension, had any major fetal abnormality, known type 2 diabetes mellitus, endocrine and hepatic disease and had any acute health condition were excluded from the study. A total 217 Participants were recruited from Feb 2012 to May 2012. Ethical clearance for the study was taken from the institutional Ethical Review Committee of National Institute of Preventive and Social Medicine (NIPSOM). After taking the written informed consent, data were collected from the participants. Respondents were interviewed ensuring the privacy and confidentiality as far as possible. Data were collected by face to face interview, document review, measurement and lab investigation. Data collected were age, parity, first visit Body Mass Inddex (BMI), prior macrosmia, bad obstetrical history, family history of diabetes mellitus (DM) and gestational diabetes mellitus (GDM). 'WHO' guidelines followed for GDM screening. Diagnosis of gestational diabetes was done by two step approach. In first step, every woman underwent $50 \mathrm{gm}$ glucose challenge test without regard to the time of day or time of last meal. This test was performed as a routine outpatient department (OPD) procedure. $50 \mathrm{gm}$ of glucose was dissolved in 200 $\mathrm{ml}$ of water and the patient was asked to drink it within 5 minutes. The time was noted and glucose level was checked exactly 1 hour after taking $50 \mathrm{gm}$ glucose, Blood drops of their lateral side of fingertips (cleaned the 1st drop and the 2nd drop was tested) were analyzed on glucometer (B. BRAUN OMNITEST Glucometer). If the glucose value at 1 hour after administration of $50 \mathrm{gm}$ glucose was $\geq 7.8$ $\mathrm{m} . \mathrm{mol} / \mathrm{l}$, a 2 hours, $75 \mathrm{gm}$ oral glucose tolerance test (OGTT) was performed within a week to confirm or rule out the presence of gestational diabetes. Blood glucose analysis for OGTT was done in the Armed Forces Institute of Pathology (AFIP) laboratory by Chemistry Analyzer machine. The OGTT was performed in the morning after 8-12 hour overnight fasting plasma glucose was determined before and 2 hour after the administration of a $75 \mathrm{~g}$ glucose containing solution. Gestational diabetes mellitus was considered to be present if the 2 hour plasma glucose value equaled or exceeded the cut-off value of $7.8 \mathrm{~m} . \mathrm{mol} / \mathrm{l}$, or if the fasting value was $>7 \mathrm{~m} . \mathrm{mol} / \mathrm{l}$, according to the World Health Organization criteria. Risk of gestational diabetes mellitus was estimated according to Chadakaran clinical prediction tool which obtained from different individual parameter including age, body mass index, family history of diabetes mellitus, birth weight of last child more than $4 \mathrm{~kg}$ and bad obstetrical history ${ }^{7}$. All the collected data were cleaned, edited and analyzed with the help of software SPSS windows version 17.0. Descriptive statistics were computed first. 
To determine the association between each variable and the occurrence of GDM $\chi^{2}$ is used for qualitative variable, independent $t$ test for continuous variable, $95 \%$ confidence intervals (95\%). A value of $p<0.05$ is considered as statistically significant. Validation of the risk score was tested on the study sample which included GDM occurrence in different risk groups, performance of the risk score according to area under curve, sensitivity, specificity, positive predictive value and negative predictive value. Variables found significantly associated with GDM in univariate analysis were considered for inclusion into the linear regression analysis to develop a new model for the study sample.

\section{Results}

During four months period, 217 respondents found eligible for the study. Three women were already diagnosed to have GDM. Therefore, $50 \mathrm{~g}$ glucose challenge test was performed on 214 women $(98.6 \%)$. Eighty three of them (38.8\%) showed an abnormal result. Oral glucose tolerance test was performed on 83 women. Out of those 21 were diagnosed as GDM resulting in a total of 24 out of 217 respondents $(11.06 \%)$ diagnosed as GDM cases. The age of the study sample ranged between 18 and 40 years with a mean \pm SD of $25.93 \pm 3.984$ years. Most of them were Muslims. Body mass index (BMI) ranged from 14.0 to 33.0 with a mean \pm SD of $23.5 \pm$ $3.52 \mathrm{~kg} / \mathrm{m}^{2}$. About $53 \%$ of the respondent had BMI within normal limit. One fourth of the respondents had diabetes in first degree relatives, $14(6.5 \%)$ had bad obstetrical history and 7 (3.2\%) gave last child birth to a baby with weight $>4 \mathrm{~kg}$ (Table-I).

Table-I: Characteristics of study population.

\begin{tabular}{|c|c|c|}
\hline Characteristics & Frequency & Percentage \\
\hline \multicolumn{3}{|l|}{ Age in years } \\
\hline$<25$ & 75 & 34.6 \\
\hline $25-30$ & 119 & 54.8 \\
\hline$>30$ & 23 & 11.6 \\
\hline MeantSD & \multicolumn{2}{|c|}{$25.93 \pm 3.984$ years } \\
\hline \multicolumn{3}{|l|}{ Body Mass Index } \\
\hline$<18.5$ & 34 & 15.7 \\
\hline $18.5-24.99$ & 115 & 53.0 \\
\hline $25.00-29.99$ & 59 & 27.2 \\
\hline$>30.00$ & 9 & 4.1 \\
\hline \multicolumn{3}{|c|}{ Bad obstetrical history(n=149) } \\
\hline No & 135 & 93.5 \\
\hline Yes & 14 & 6.5 \\
\hline \multicolumn{3}{|c|}{ DM in first degree relatives } \\
\hline No & 162 & 74.7 \\
\hline yes & 55 & 25.3 \\
\hline \multicolumn{3}{|c|}{ Last child birth weight $>4 \mathrm{~kg}$} \\
\hline No & 210 & 96.8 \\
\hline Yes & 7 & 3.2 \\
\hline
\end{tabular}

JAFMC Bangladesh. Vol 11, No 1 (June) 2015
According to Chadakaran clinical prediction tool Individual risk score was calculated from the equation - Risk score $=6$ age $+11 \mathrm{BMI}+109$ family history of diabetes +42 prior macrosomia +49 bad obstetrical history. The range of estimated risk score ranged from 293 to 735 with a mean \pm SD of $446.2 \pm 75.9$. By using 380 and 460 as cutoff points respondents were categorized into three groups: Score below 380 was categorized into low risk, above 460 categorized as high risk and in between of 380 and 460 categorized as intermediate risk group (Fig-1).

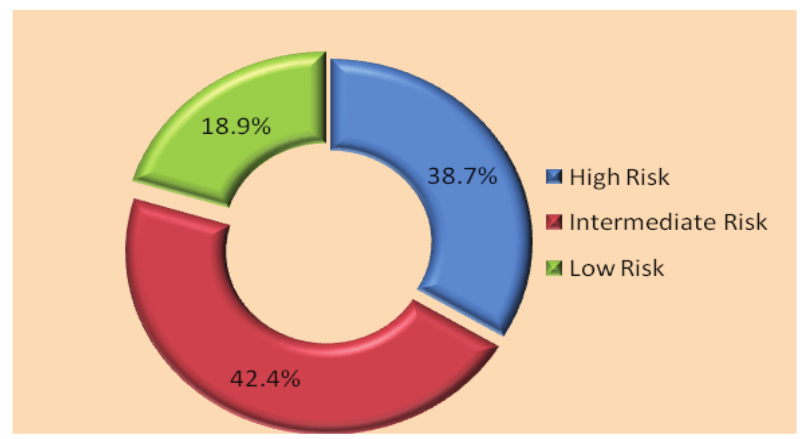

Fig-1: Risk of GDM of respondents.

Performances of the risk score to predict GDM in the study population shown in the table 2 . At the level of $\geq 380$, sensitivity was $100 \%$ and specificity $21.8 \%$, positive predictive value $13.63 \%$ and negative predictive value $100 \%$ and area under curve 0.380 . At the level of 460 score the sensitivity and specificity were found closest $(70.8 \%$ and $65.3 \%)$, positive predictive value and negative predictive value was found as, $20.23 \%$ and $94.73 \%$ respectively. Because of the screening purpose of the risk score, the cut off value was determined by giving high priority to the sensitivity than specificity. When the risk equation was tested among the study population, the ROC curve of the risk score also demonstrated good function to predict GDM, with an area under curve $0.763(95 \%, 0.682-0.845)$ (Figure-2).

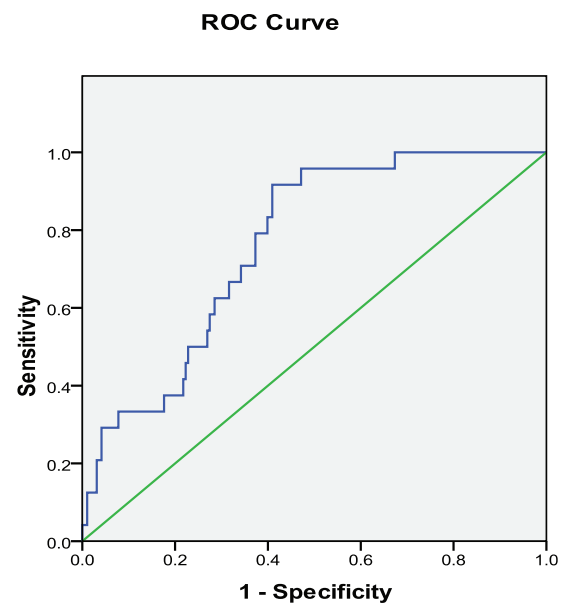


Table-II: Performances of risk score at different cut off to predict GDM.

\begin{tabular}{|c|c|c|c|c|c|c|}
\hline $\begin{array}{c}\text { Cutoff } \\
\text { score? }\end{array}$ & $\begin{array}{c}\text { Sensitivity } \\
\%\end{array}$ & $\begin{array}{c}\text { Specificity } \\
\%\end{array}$ & $\begin{array}{c}\text { Positive } \\
\text { predictive } \\
\text { value } \%\end{array}$ & $\begin{array}{c}\text { Negative } \\
\text { predictive } \\
\text { value } \%\end{array}$ & $\begin{array}{c}\text { Number of GDM } \\
\text { with false-negative } \\
\text { test (\%) }\end{array}$ & AUC \\
\hline 380 & 100.0 & 21.8 & 13.6 & 100.0 & $0(0)$ & 0.385 \\
\hline 400 & 95.8 & 33.7 & 15.2 & 96.1 & $1(4.1)$ & 0.478 \\
\hline 420 & 95.8 & 44.6 & 17.5 & 98.8 & $1(4.1)$ & 0.407 \\
\hline 440 & 91.7 & 54.9 & 20.0 & 98.1 & $2(8.33)$ & 0.529 \\
\hline 460 & 70.8 & 65.3 & 20.2 & 94.7 & $7(29.16)$ & 0.657 \\
\hline 480 & 58.3 & 72.5 & 20.9 & 93.3 & $10(41.66)$ & 0.597 \\
\hline 500 & 41.7 & 77.7 & 15.8 & 90.9 & $14(58.33)$ & 0.574 \\
\hline 520 & 33.3 & 82.9 & 19.0 & 90.8 & $16(66.66)$ & 0.548 \\
\hline 540 & 33.3 & 90.7 & 32.0 & 91.6 & $16(66.66)$ & 0.480 \\
\hline 560 & 25.0 & 95.9 & 42.8 & 91.1 & $18(75)$ & 0.408 \\
\hline 580 & 12.5 & 97.4 & 37.5 & 89.9 & $21(87.5)$ & 0.629 \\
\hline
\end{tabular}

Highest proportion of GDM found with high risk category 17(20.2\%). In intermediate risk group 7 (7.6\%) respondents found with GDM. None of the low risk group developed GDM. Significant association was observed between clinically predicted risk and occurrence of GDM $(p<0.001)$. Significant association was observed between clinically predicted risk and occurrence of GDM $(p<0.001)$ (Table-III).

Table-III: Occurrence of GDM in respondents of different risk group.

\begin{tabular}{|c|c|c|c|c|c|}
\hline Risk of GDM & Non GDM (\%) & GDM (\%) & Total & $\chi^{2}$ & $\mathbf{p}$ \\
\hline Low risk & $41(100)$ & $0(0)$ & 41(18.9) & \multirow{3}{*}{$13.750^{* *}$} & \multirow{3}{*}{0.001} \\
\hline Intermediate risk & $85(92.4)$ & $7(7.6)$ & $92(42.4)$ & & \\
\hline High risk & $67(79.8)$ & $17(20.2)$ & $84(38.7)$ & & \\
\hline
\end{tabular}

\section{Discussion}

Increasing trends of gestational diabetes mellitus (GDM) all over the world particularly in low and middle income countries impose a variety of health policy, health service delivery and technical challenges across the spectrum of worldwide healthcare system. To lessen the number of unnecessary glucose challenge tests, several authors have developed clinical scoring prediction model to provide an estimation of the risk of GDM for each individual pregnant woman. Chadakaran's clinical prediction model was used in this study to estimate the risk of GDM in pregnant women ${ }^{7}$. It was developed and validated among the Thai women in Bangkok Metropolitan Administration Medical College and Vajira Hospital, Bangkok, Thailand. The model included five basic clinical data which can be readily obtained from pregnant women such as age, BMI as continuous variables, first trimester BMI, bad obstetrical history and family history of diabetes disregarding the ethnic factor in determining the risk for GDM and the score obtained by the equation (Risk score $=6 a g e+11 \mathrm{BMI}+109$ family history of diabetes +42 prior macrosmia +49 bad obstetrical history). Another clinical scoring system was developed by Naylor. A clinical scoring system based on age, body mass index and ethnicity was developed from a prospective cohort study in Toronto Trihospital (Toronto hospital, Mt.Sianai hospital and universityof Toronto,Canada). The selective screening strategy based on this scoring system showed that the number of screening and diagnostic tests could be decreased for comparable detection rates ${ }^{8}$. A limitation of the study is that they did not include the women younger than 24 years so that it cannot be used in those countries of younger maternal age such as Bangladesh. Another potential disadvantage is tagging high risk for Asian race. More recently, Caliskan formed a risk factor score using five variables: Maternal age $\geq 25$ years, body mass index $\geq 25 \mathrm{~kg} / \mathrm{m}^{2}$, family history of diabetes, prior macrosmia, and adverse outcome during pregnancies $^{9}$. Application of this risk scoring system to the general population with younger maternal age such 
as Bangladesh might yield lesser benefit. Moreover influence of ethnicity and categorical scoring of age and BMI limit the ability to generalize risk score across populations. Due to have similar population, similar risk of Asian race, including all age group and basic five clinical data which can be readily obtained from pregnant women Chadakaran's clinical prediction tool was used for this study. Considering the diagnostic performances to predict women with GDM it can be said that the model is effective in our country perspective though it has some limitation such as the strategy was to avoid the screening if a risk scores below 380 resulting only $18.9 \%$ reduction in the number of women requiring GCT or OGTT without any significant decrease in GDM detection, whereas in Chadakaran study $41.3 \%$ of women could avoid GCT. As a general practice, we performed the diagnostic OGTT only in individuals with an abnormal GCT, so some women who had normal GCT but actually was GDM would have been missed. So this score can not used for diagnostic purpose of GDM or can not replace GCT or OGTT.

\section{Conclusion}

This cross sectional study was an attempt to develop an accurate clinical prediction model that facilitates clinician to estimate the probability of GDM clinically at first antenatal visit irrespective of duration of pregnancy and place of antenatal check up rural or urban so that further testing and monitoring of women can be individualized on the basis of this probability, allowing for timely intervention to improve maternal and neonatal health and also to save time as well as health care cost.

Chadakaran prediction model was found an effective screening tool in predicting women who are likely to develop GDM in this study. All the women with GDM in this study were detected using this model but simultaneously more than two third of the sample felt under either intermediate or high risk category. Use of clinical prediction model is a simple, non invasive method based on readily available data can be easily introduced for screening of gestational diabetes as a part of the standard antenatal care package. To evaluate the external validity of the findings of this study before clinical practice, further study with large sample size in different population is required.

\section{References}

1. Sayeeda MA, Mahtab $\mathrm{H}$, Khanam PA et al. Diabetes and HTN in pregnancy in a rural community of Bangladesh: A population based study. Diabet Med 2005; 22 (7):1267-71.

2. Ferrara A, Kahn HS, Quesenberry CP et al. An increase in the incidence of gestational diabetes mellitus; Northern California: 1991-2000. Obstet Gynecol 2004; 103: 526-33.

3. Rumbold AR, Crowther CA. Guideline use for gestational diabetes mellitus and current screening, diagnostic and management practices in Australian hospitals. Aust NZJ Obstet Gynaecol 2001;41: 86-90.

4. Langer $\mathrm{O}$, Rodriguez DA, Xenakis EM et al. Intensified versus conventional management of gestational diabetes. Am J Obstet Gynecol 1994; 170:1642-7.

5. Brody SC, Harris R, Lohr K. Screening for gestational diabetes: a summary of the evidence for the U.S. Preventive Services Task Force. Obstet Gynecol 2003; 101:380-92.

6. United Nations Department for economic and social information and policy analysis. World population prospects: the 2004 revision. New York: United Nations; 2005.

7. Phaloprakarn C, Tangjitgamo S, Manusirivithaya S. A risk scores for selective screening of gestational diabetes mellitus. European Journal of Obstetrics \& Gynecology and Reproductive Biology 145 (2009);145:71-5.

8. Naylor D, Sermer M, Chen E et al. Selective screening for gestational diabetes mellitus. The New England Journal of Medicine, 1997; 337:1591-96.

9. Caliskan E, Kayikcioglu F, Ozturk $\mathrm{N}$ et al. A population based risk factor scoring will decrease unnecessary testing for the diagnosis of gestational diabetes mellitus. Acta Obstet Gynecol Scand 2004; 83: $524-30$. 